\title{
Asymptotic Behaviour of the QED Perturbation Series
}

\author{
Idrish Huet, ${ }^{1}$ Michel Rausch de Traubenberg, ${ }^{2}$ and Christian Schubert ${ }^{3}$ \\ ${ }^{1}$ Facultad de Ciencias en Física y Matemáticas, Universidad Autónoma de Chiapas, Ciudad Universitaria, \\ 29050 Tuxtla Gutiérrez, CHIS, Mexico \\ ${ }^{2}$ IPHC-DRS, UdS, IN2P3, 23 rue du Loess, 67037 Strasbourg Cedex, France \\ ${ }^{3}$ Instituto de Física y Matemáticas, Universidad Michoacana de San Nicolás de Hidalgo, Edificio C-3, \\ Apdo. Postal 2-82, 58040 Morelia, MICH, Mexico \\ Correspondence should be addressed to Christian Schubert; schubert@ifm.umich.mx
}

Received 23 April 2017; Accepted 12 July 2017; Published 19 September 2017

Academic Editor: Ralf Hofmann

Copyright (C) 2017 Idrish Huet et al. This is an open access article distributed under the Creative Commons Attribution License, which permits unrestricted use, distribution, and reproduction in any medium, provided the original work is properly cited. The publication of this article was funded by SCOAP ${ }^{3}$.

\begin{abstract}
I will summarize the present state of a long-term effort to obtain information on the large-order asymptotic behaviour of the QED perturbation series through the effective action. Starting with the constant-field case, I will discuss the Euler-Heisenberg Lagrangian in various dimensions and up to the three-loop level. This Lagrangian holds the information on the N-photon amplitudes in the lowenergy limit, and combining it with Spinor helicity methods explicit all-N results can be obtained at the one-loop and, for the "all +" amplitudes, also at the two-loop level. For the imaginary part of the Euler-Heisenberg Lagrangian, an all-loop formula has been conjectured independently by Affleck, Alvarez, and Manton for Scalar QED and by Lebedev and Ritus for Spinor QED. This formula can be related through a Borel dispersion relation to the leading large- $\mathrm{N}$ behaviour of the $\mathrm{N}$-photon amplitudes. It is analytic in the fine structure constant, which is puzzling and suggests a diagrammatic investigation of the large- $\mathrm{N}$ limit in perturbation theory. Preliminary results of such a study for the $1+1$ dimensional case throw doubt on the validity of the conjecture.
\end{abstract}

\section{Motivation}

In 1952 Dyson [1] shocked the high energy physics community by declaring that, quite generally, the QED perturbation series cannot converge. Writing the series as

$$
F\left(e^{2}\right)=c_{0}+c_{2} e^{2}+c_{4} e^{4}+\cdots,
$$

Dyson argues, "suppose, if possible, that the series converges for some positive value of $e^{2}$; this implies that $F\left(e^{2}\right)$ is an analytic function of $e$ at $e=0$. Then for sufficiently small values of $e, F\left(-e^{2}\right)$ will also be a well-behaved analytic function with a convergent power-series expansion."

He then argues that, on physical grounds, this cannot be the case, since for $e^{2}<0$ the QED vacuum will be unstable due to a runaway production of $e^{+} e^{-}$pairs which coalesce into like-charge groups.

Shortly later Hurst [2] already provided a mathematical proof of this fact for scalar $\lambda \phi^{3}$ theory. The proof is essentially based on the following three elements.
(1) The use of the inequality

$$
\prod_{i=1}^{F}\left(\frac{1}{p_{i}^{2}+\kappa^{2}}\right) \geq \frac{F^{F}}{\left(\sum_{i=1}^{F} p_{i}^{2}+F \kappa^{2}\right)^{F}}
$$

to establish lower bounds for arbitrary Feynman diagrams (in the Euclidean).

(2) Proof that the number of distinct Feynman diagrams at $n$th loop order grows like $(n / 2) ! n !$

(3) Absence of sign cancellations between graphs.

In 1994, 't Hooft [3] found another very general, but very different, argument against convergence of the perturbation series based on renormalon chains. Thus today it is believed that the perturbation series in nontrivial quantum field theories generically is asymptotical rather than convergent, so that summation methods must be used. Of those by far the most important one is Borel summation, since it is ideally suited to the typical factorial growth of perturbation 
theory coefficients. Let me remind you that, for a factorially divergent series

$$
F(g) \sim \sum_{n=0}^{\infty} c_{n} g^{n+1}
$$

one defines the Borel transform as

$$
B(t) \equiv \sum_{n=0}^{\infty} c_{n} \frac{t^{n}}{n !} .
$$

If $B(t)$ has no singularities on the positive real axis and does not increase too rapidly at infinity, one can also define the Borel integral

$$
\widetilde{F}(g) \equiv \int_{0}^{\infty} d t e^{-t / g} B(t) .
$$

$\widetilde{F}$ is the Borel sum of the original series $F . F$ is asymptotic to $\widetilde{F}$ by construction, although the true physical quantity represented by the series $F$ might still differ from $\widetilde{F}$ by nonperturbative terms. The Borel transform remains a useful concept even when it leads to singularities, since those usually contain information on the large-order structure of the theory. In many cases they can be traced either to instantons, renormalons, or Euclidean bounces.

Until recently, there was a dearth of nontrivial examples for field theory models where sufficient information would be available to decide the question of Borel summability in a definite manner. Fortunately, this has changed through the advent of supersymmetry; in recent years Borel summability (or Borel nonsummability) has been rigorously demonstrated in a number of supersymmetric models [4].

Even when Borel summability does not apply, Borel analysis can still be very useful through the use of Borel dispersion relations. This goes as follows. Assume that a function $F(g)$ has an asymptotic series expansion

$$
F(g) \sim \sum_{n=0}^{\infty} c_{n} g^{n}
$$

where the expansion coefficients $c_{n}$ have the leading-order large $n$ behaviour

$$
c_{n} \sim \rho^{n} \Gamma(\mu n+\nu)
$$

with some real constants $\rho>0, \mu>0$, and $\nu$. It is easy to see that such a series is not Borel-summable, since the Borel integral (5) can never converge (e.g., in the textbook case $\mu=\nu=1$ it has a pole at $t=1 / \rho$ ). Nevertheless, applying a dispersion relation to this integral one can show that the leading contribution to its imaginary part for small $g$ is given by

$$
\operatorname{Im} F(g) \sim \frac{\pi}{\mu}\left(\frac{1}{\rho g}\right)^{v / \mu} \exp \left[-\left(\frac{1}{\rho g}\right)^{1 / \mu}\right] .
$$

Coming back to the case of QED, given the arguments by Dyson and ' $t$ Hooft it is certainly safe to exclude a nonzero convergence radius of the full QED perturbation series. However, despite the immense work that has gone into loworder perturbative QED computations, presently still little is known about the precise large-order behaviour of the coefficients. Contrary to the case of scalar field theories mentioned above, straightforward estimates based on lower bounds for individual diagrams cannot be used in gauge theory, since here Feynman diagrams come with different signs, and gauge invariance is known to lead to cancellations between them. And these cancellations are particularly extensive in the abelian case, where there are no obstructing color factors. Thus QED in this respect is more difficult than QCD, which is made worse by the absence of (space-time) instantons in QED, which in the nonabelian case can provide some large-order information. In 1977 Cvitanovic [5] suggested, based on an analysis of the calculation of the three-loop anomalous magnetic momentum $g-2$ which he had done with Cvitanovic and Kinoshita [6], that these cancellations should be taken into account by counting the number of classes of gauge-invariant diagrams, rather than the number of individual diagrams. He also conjectured that, for the case of $g-2$, they reduce the coefficients of the perturbation series sufficiently to make it convergent in the quenched approximation. This conjecture, though nowadays forgotten, is actually still standing, since neither Dyson's nor ' $t$ Hooft's arguments work in the absence of fermionic bubbles.

Here I will summarize the state of a long-term effort [7-15] to get information on the large-order behaviour of the QED perturbation series using the Euler-Heisenberg Lagrangian and its higher-loop radiative corrections.

\section{The 1-Loop Euler-Heisenberg Lagrangian}

The Euler-Heisenberg Lagrangian ("EHL") is the one-loop QED effective Lagrangian for a constant external field. Heisenberg and Euler [16] obtained for it in 1936 the following well-known proper-time representation:

$$
\begin{aligned}
& \mathscr{L}_{\text {spin }}^{(1)}(F)=-\frac{1}{8 \pi^{2}} \int_{0}^{\infty} \frac{d T}{T^{3}} \mathrm{e}^{-m^{2} T} \\
& \cdot\left[\frac{(e a T)(e b T)}{\tanh (e a T) \tan (e b T)}-\frac{e^{2}}{3}\left(a^{2}-b^{2}\right) T^{2}-1\right] .
\end{aligned}
$$

Here $a, b$ are the two invariants of the Maxwell field, related to $\mathbf{E}, \mathbf{B}$ by

$$
a^{2}-b^{2}=B^{2}-E^{2}, \quad a b=\mathbf{E} \cdot \mathbf{B} .
$$

The analogous result for Scalar QED was obtained by Weisskopf [17] but will be called "Scalar Euler-Heisenberg Lagrangian" in the following:

$$
\begin{aligned}
& \mathscr{L}_{\text {scal }}^{(1)}(F)=\frac{1}{16 \pi^{2}} \int_{0}^{\infty} \frac{d T}{T^{3}} \mathrm{e}^{-m^{2} T}\left[\frac{(e a T)(e b T)}{\sinh (e a T) \sin (e b T)}\right. \\
& \left.+\frac{e^{2}}{6}\left(a^{2}-b^{2}\right) T^{2}-1\right] .
\end{aligned}
$$

The Euler-Heisenberg Lagrangian ("EHL”) holds information on the one-loop $\mathrm{N}$-photon amplitudes, but only in the 


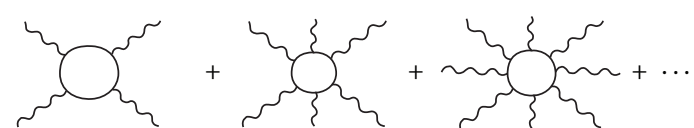

FIgURE 1: Sum of diagrams equivalent to the one-loop EHL.

low-energy limit (since a constant field can emit only zeroenergy photons).

Thus diagrammatically $\mathscr{L}^{(1)}(F)$ is equivalent to the sum of the Feynman graphs shown in Figure 1, where all photon energies are small compared to the electron mass, $\omega_{i} \ll m$.

In [10] it was shown how to carry out explicitly the construction of these limiting low-energy amplitudes starting from the weak-field expansion of the EHL:

$$
\mathscr{L}(F)=\sum_{k, l} c_{k l} a^{2 k} b^{2 l} .
$$

It turned out that if one fixes the number of photons, their momenta $k_{1}, \ldots, k_{N}$, and a helicity assignment for each photon, then in this limit the full dependence on the momenta and polarization vectors is carried by a unique invariant. Thus the magnitude of the amplitude can be specified by a single number, which will be essential for our whole approach.

Except for the purely magnetic case, the EHL has also an imaginary part related to vacuum pair creation by the electric field component (to be called "Sauter-Schwinger pair creation" in the following) $[18,19]$. In the purely electric case one finds, from the poles in the $T$-integration, the following decomposition due to Schwinger [19]:

$$
\begin{aligned}
& \operatorname{Im} \mathscr{L}^{(1)}(E)=\frac{m^{4}}{8 \pi^{3}} \beta^{2} \sum_{k=1}^{\infty} \frac{1}{k^{2}} \exp \left[-\frac{\pi k}{\beta}\right], \\
& \operatorname{Im} \mathscr{L}_{\text {scal }}^{(1)}(E)=-\frac{m^{4}}{16 \pi^{3}} \beta^{2} \sum_{k=1}^{\infty} \frac{(-1)^{k}}{k^{2}} \exp \left[-\frac{\pi k}{\beta}\right] .
\end{aligned}
$$

$\left(\beta=e E / m^{2}\right)$. In the following we will focus on the weakfield limit $\beta \ll 1$ where only the first of these "Schwingerexponentials" is relevant.

The nonperturbative dependence of the Schwingerexponentials on the field supports the interpretation of field-induced pair creation as a vacuum tunneling effect, as proposed by Sauter as early as 1931 [18].

As usual in quantum field theory, the real and imaginary parts of the EHL are related by a dispersion relation. For the $N$-photon amplitudes at full momentum, this would be a standard dispersion relation performed diagram-by-diagram, relating the diagrams of Figure 1 to the "cut diagrams" shown in Figure 2, involving on-shell electrons.

However, in the zero-energy limit the cut diagrams all vanish, since a finite number of zero-energy photons cannot create a pair on-shell. Thus what counts here is only the asymptotic behaviour for a large number of photons, and instead of an ordinary dispersion relation we have to use a Borel dispersion relation. This works in the following way [7].

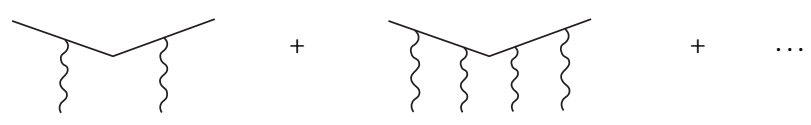

FIgURE 2: Cut diagrams giving the imaginary part of the $N$-photon amplitudes.

Consider the purely magnetic EHL. Expanding it out in powers of the field yields

$$
\begin{aligned}
& \mathscr{L}^{(1)}(B) \\
& =-\frac{1}{8 \pi^{2}} \int_{0}^{\infty} \frac{d T}{T^{3}} \mathrm{e}^{-m^{2} T}\left[\frac{e B T}{\tanh (e B T)}-\frac{1}{3}(e B T)^{2}-1\right] \\
& =\frac{2 m^{4}}{\pi^{2}} \sum_{n=2}^{\infty} c_{n}^{(1)} g^{n}
\end{aligned}
$$

with an effective expansion parameter $g=\left(e B / m^{2}\right)^{2}$, and coefficients $c_{n}^{(1)}$ that can be written in terms of the Bernoulli numbers $B_{n}$ :

$$
c_{n}^{(1)}=-\frac{2^{2 n-4} B_{2 n}}{(2 n)(2 n-1)(2 n-2)} .
$$

Here $c_{n}^{(1)}$ holds information on the $N=2 n$ photon amplitudes. The asymptotic behaviour of the coefficients can be easily studied using well-known properties of the Bernoulli numbers. One finds

$$
c_{n}^{(1)} \stackrel{n \rightarrow \infty}{\sim}(-1)^{n} \frac{1}{8} \frac{\Gamma(2 n-2)}{\pi^{2 n}}\left(1+\frac{1}{2^{2 n}}+\frac{1}{3^{2 n}}+\cdots\right) .
$$

Thanks to the factor $(-1)^{n}$, the individual terms on the right hand side of (16) all give convergent Borel integrals. This is one (rather roundabout) way of seeing that the purely magnetic EHL has no imaginary part and does not give rise to pair creation.

The analogous expansion for the purely electric field case is almost the same:

$$
\begin{aligned}
& \mathscr{L}^{(1)}(E) \\
& =-\frac{1}{8 \pi^{2}} \int_{0}^{\infty} \frac{d T}{T^{3}} \mathrm{e}^{-m^{2} T}\left[\frac{e E T}{\tan (e E T)}+\frac{1}{3}(e E T)^{2}-1\right] \\
& =\frac{2 m^{4}}{\pi^{2}} \sum_{n=2}^{\infty}(-1)^{n} c_{n}^{(1)} g^{n},
\end{aligned}
$$

where now $g=\left(e E / m^{2}\right)^{2}$, but with the same $c_{n}^{(1)}$. However, the additional factor $(-1)^{n}$ makes the series nonalternating, which is crucial, because now the termwise use of expansion (16) leads to divergent Borel integrals. These divergent integrals do, however, all possess well-defined imaginary parts, by a (now ordinary) dispersion relation. One finds 
a perfect match between expansion (16) and Schwinger's expansion (13):

$$
\begin{gathered}
c_{n}^{(1)} \sim(-1)^{n} \frac{1}{8} \frac{\Gamma(2 n-2)}{\pi^{2 n}} \longrightarrow \operatorname{Im} \mathscr{L}^{(1)}(E) \sim \frac{m^{4}}{8 \pi^{3}}\left(\frac{e E}{m^{2}}\right)^{2} \exp \left(-\frac{\pi m^{2}}{e E}\right), \\
c_{n}^{(1)} \sim(-1)^{n} \frac{1}{8} \frac{\Gamma(2 n-2)}{\pi^{2 n}} \frac{1}{2^{2 n}} \longrightarrow \operatorname{Im} \mathscr{L}^{(1)}(E) \sim \frac{m^{4}}{8 \pi^{3}}\left(\frac{e E}{m^{2}}\right)^{2} \frac{1}{2^{2}} \exp \left(-2 \frac{\pi m^{2}}{e E}\right),
\end{gathered}
$$

\section{The Euler-Heisenberg Lagrangian at} Higher Loops

Proceeding to higher-loop orders, the two-loop EHL $\mathscr{L}^{(2)}$ is generated by the diagrams shown in Figure 3 (here and in the following it is understood that internal photon corrections are put in all possible ways).

As in the one-loop case, $\mathscr{L}^{(2)}$ will have an imaginary part iff the field is not purely magnetic. This imaginary part corresponds to the one-loop "cut diagrams" depicted in Figure 4.

The two-loop EHL was first studied by Ritus, both for Spinor [20] and for Scalar QED [21]. These calculations, as well as later recalculations $[12,22,23]$, resulted in a type of rather intractable two-parameter integrals. However, the first few coefficients of the weak-field expansions of the two-loop EHLs have been computed [7, 12, 23]. As to the imaginary parts, the Schwinger formulas (13) generalize to the two-loop level as follows [24]:

$$
\begin{aligned}
& \operatorname{Im} \mathscr{L}^{(2)}(E)=\frac{m^{4}}{8 \pi^{3}} \beta^{2} \sum_{k=1}^{\infty} \alpha \pi K_{k}^{\text {spin }}(\beta) \exp \left[-\frac{\pi k}{\beta}\right], \\
& \operatorname{Im} \mathscr{L}_{\text {scal }}^{(2)}(E) \\
& \quad=\frac{m^{4}}{16 \pi^{3}} \beta^{2} \sum_{k=1}^{\infty}(-1)^{k+1} \alpha \pi K_{k}^{\text {scal }}(\beta) \exp \left[-\frac{\pi k}{\beta}\right] .
\end{aligned}
$$

$\left(\alpha=e^{2} / 4 \pi\right)$, where

$$
\begin{aligned}
K_{k}^{\text {scal,spin }}(\beta) & =-\frac{c_{k}}{\sqrt{\beta}}+1+\mathrm{O}(\sqrt{\beta}) \\
c_{k} & =\frac{1}{2 \sqrt{k}} \sum_{l=1}^{k-1} \frac{1}{\sqrt{l(k-l)}}, \quad k \geq 2, \quad c_{1}=0
\end{aligned}
$$

(these coefficients, also called $c_{n}$, should not be confused with the weak-field expansion coefficients introduced above). Thus at two loops the $k$ th Schwinger-exponential appears with a prefactor which is still a function of the field strength, of which presently only the lowest order terms in the weakfield expansion are known. Still, things become very simple at leading order in this expansion: adding the one-loop and two-loop EHLs, one finds, for example, for the Spinor QED case [24],

$$
\operatorname{Im} \mathscr{L}^{(1)}(E)+\operatorname{Im} \mathscr{L}^{(2)}(E) \stackrel{\beta \rightarrow 0}{\sim} \frac{m^{4} \beta^{2}}{8 \pi^{3}}(1+\alpha \pi) \mathrm{e}^{-\pi / \beta},
$$

and this result is spin-independent (but for the normalization). In [24] it was further noted that if one assumes that in this weak-field approximation higher order corrections just lead to an exponentiation

$$
\sum_{l=1}^{\infty} \operatorname{Im} \mathscr{L}^{(l)}(E) \stackrel{\beta \rightarrow 0}{\sim} \operatorname{Im} \mathscr{L}^{(1)}(E) \mathrm{e}^{\alpha \pi}
$$

then the $\mathrm{e}^{\alpha \pi}$ factor can be absorbed into the Schwinger factor $\mathrm{e}^{-\pi / \beta}$ by the following mass-shift:

$$
m(E) \approx m-\frac{\alpha}{2} \frac{e E}{m} .
$$

Moreover, the existence of this mass-shift can be independently confirmed in two different ways. First, the same massshift had been found by Ritus already before in the crossed process of electron propagation in the electric field [25]. Second, in the tunneling picture it can be interpreted as the correction to the Schwinger pair creation rate due to the pair being created with a negative Coulomb interaction energy at a definite distance, taking the Coulomb interaction into account at the one-photon exchange level [24].

And although this was not known to the authors of [24], an analogous exponentiation had already been conjectured two years before for the Scalar QED case by Affleck et al. [26]. However, those authors used a totally different approach based on worldline instantons. To explain this concept, we first have to discuss Feynman's worldline path integral representation of the QED effective action.

\section{Worldline Representation of the QED Effective Action}

In 1950 Feynman presented, in an appendix to one of his groundbreaking papers on the modern, manifestly relativistic formalism of QED [27], also an alternative first-quantized formulation of Scalar QED, "for its own interest as an alternative to the formulation of second quantization." There he provides a simple rule for constructing the complete Scalar 


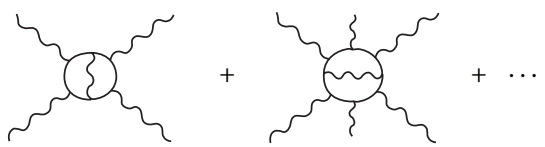

FIgURE 3: Feynman diagrams contributing to the 2-loop EHL.

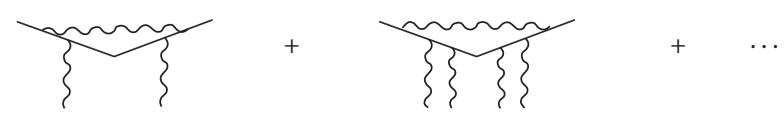

FIGURE 4: Feynman diagrams contributing to 2-loop Schwinger pair creation.

QED S-matrix by representing the scalar particles in terms of relativistic particle path integrals, and coupling them through photons in all possible ways. Upon restriction to the purely photonic part of the $S$-matrix (no external scalars) and to the "quenched" contribution (only one virtual scalar), this "worldline representation" can be stated very compactly in terms of the (quenched) effective action $\Gamma[A]$ :

$$
\begin{aligned}
\Gamma_{\text {scalar }}[A] & =\int d^{4} x \mathscr{L}_{\text {scalar }}[A] \\
& =\int_{0}^{\infty} \frac{d T}{T} \mathrm{e}^{-m^{2} T} \int_{x(T)=x(0)} \mathscr{D} x(\tau) e^{-S[x(\tau)]} .
\end{aligned}
$$

Here $T$ denotes the proper-time of the scalar particle in the loop, $m$ its mass, and $\int_{x(T)=x(0)} \mathscr{D} x(\tau)$ a path integral over all closed loops in space-time with fixed periodicity in the proper-time. The worldline action $S[x(\tau)]$ has three parts:

$$
S=S_{0}+S_{\text {ext }}+S_{\text {int }}
$$

They are given by

$$
\begin{aligned}
& S_{0}=\int_{0}^{T} d \tau \frac{\dot{x}^{2}}{4} \quad \text { (free propagation) }, \\
& S_{\mathrm{ext}}=i e \int_{0}^{T} \dot{x}^{\mu} A_{\mu}(x(\tau)) \quad(\text { external photons), } \\
& S_{\mathrm{int}}=-\frac{e^{2}}{8 \pi^{2}} \int_{0}^{T} d \tau_{1} \int_{0}^{T} d \tau_{2} \frac{\dot{x}\left(\tau_{1}\right) \cdot \dot{x}\left(\tau_{2}\right)}{\left(x\left(\tau_{1}\right)-x\left(\tau_{2}\right)\right)^{2}} \\
& \text { (internal photons). }
\end{aligned}
$$

The kinetic term $S_{0}$ describes the free propagation of the scalar, $S_{\text {ext }}$ describes its interaction with the external field, and $S_{\text {int }}$ generates the corrections due to internal photon exchanges in the loop. Expanding out the two interaction exponentials leads back to Feynman diagrams, however with the important difference that no particular ordering of the photon legs along the loop needs to be fixed. Thus the term $S_{\text {ext }}$ alone upon expansion yields the diagrams of Figure 1 (where each leg now stands for an interaction with the arbitrary field $A(x))$.

The "worldline instanton" of Affleck et al. [26] is an extremal trajectory of the worldline path integral for a stationary phase approximation. For the case of a constant electric field in the $z$ direction this extremal action trajectory is given by a circle in the (Euclidean) $t-z$ plane:

$$
x_{\text {instanton }}(\tau)=\frac{m}{e E}\left(0,0, \cos \left(\frac{2 \pi \tau}{T}\right), \sin \left(\frac{2 \pi \tau}{T}\right)\right) .
$$

It can be shown that in the weak-field (= large mass) limit the imaginary (although not the real) part of the effective Lagrangian can be well-approximated by replacing the path integral with this single trajectory:

$$
\operatorname{Im} \mathscr{L}_{\text {scalar }}^{\text {(quenched) }}(E) \sim \mathrm{e}^{-S\left[x_{\text {instanton }}\right]} .
$$

This is easily evaluated to be

$$
\begin{aligned}
\left(S_{0}+S_{\text {ext }}\right)\left[x_{\text {instanton }}\right] & =\pi \frac{m^{2}}{e E}, \\
S_{\text {int }}\left[x_{\text {instanton }}\right] & =-\alpha \pi
\end{aligned}
$$

Thus the contribution of $S_{0}+S_{\text {ext }}$ just reproduces the leading (one-loop) Schwinger-exponential of (13) and the one of $S_{\text {int }}$ the $\mathrm{e}^{\alpha \pi}$ factor.

Thus Affleck et al. arrive, with very little effort, at the same exponentiation for Scalar QED that Lebedev and Ritus find in Spinor QED:

$$
\begin{aligned}
\operatorname{Im} \mathscr{L}_{\text {scal }}^{(\text {all-loop) }}(E) & =\sum_{l=1}^{\infty} \operatorname{Im} \mathscr{L}_{\text {scal }}^{(l)}(E) \\
& \stackrel{\beta \rightarrow 0}{\sim}-\frac{m^{4} \beta^{2}}{16 \pi^{3}} \exp \left[-\frac{\pi}{\beta}+\alpha \pi\right] \\
& =\operatorname{Im} \mathscr{L}_{\text {scal }}^{(1)}(E) \mathrm{e}^{\alpha \pi} .
\end{aligned}
$$

Their argument assumes the field to be weak, but there is no restriction on the strength of the coupling $\alpha$. We note the following:

(i) Formula (30), if true, constitutes a rare case of an all-loop summation of an infinite series of graphs of arbitrary loop order. Those graphs are shown in Table 1.

(ii) According to [26], the contribution of all nonquenched diagrams gets suppressed in the weak-field limit.

(iii) Perhaps, most surprisingly, the scalar mass appearing in (30) is already the physically renormalized one, implying that the worldline instanton approach automatically takes all mass renormalization counterdiagrams into account. This is remarkable considering that the determination of the physical mass parameter for the EHL becomes a rather nontrivial issue already at two loops [20, 22, 23].

Thus, according to Affleck et al. a true all-loop summation has produced the factor $\mathrm{e}^{\alpha \pi}$, which is not only unreasonably simple but also perfectly analytical in the fine structure constant $\alpha$ !. According to what has been said above, this 
TABLE 1: Feynman diagrams contributing to the AAM formula.

Number of loops

would seem to point towards extensive cancellations between Feynman diagrams. However, neither [26] nor [24] made this point, perhaps because the Schwinger pair creation rate is a rather peculiar quantity. Thus our next goal will be to transfer the exponential factor from the imaginary to the real part of the EHL, by a Borel dispersion relation, and from there to the low-energy photon $S$-matrix through the same procedure as at one-loop:

$$
\operatorname{Im} \mathscr{L} \stackrel{\text { disp. rel. }}{\longrightarrow} \operatorname{Re} \mathscr{L} \longrightarrow \Gamma\left[k_{1}, \varepsilon_{1} ; \ldots ; k_{N}, \varepsilon_{N}\right] .
$$

First we need to see whether our one-loop Borel dispersion relation can be extended to the multiloop level. For this it will be useful to consider the simplest possible nonzero constant-field background, which is the self-dual one.

\section{The Self-Dual Case}

As we mentioned above, the two-loop correction to the EHL for a purely electric or purely magnetic field are known only in terms of intractable integrals, and only the first few coefficients have been calculated so far. However, this case is not the simplest one that one can consider; mathematically much better behaved is the one of a (Euclidean) constant selfdual field, defined by $F_{\mu \nu}=(1 / 2) \varepsilon_{\mu \nu \alpha \beta} F^{\alpha \beta}$. The field strength tensor can be written as

$$
F=\left(\begin{array}{cccc}
0 & f & 0 & 0 \\
-f & 0 & 0 & 0 \\
0 & 0 & 0 & f \\
0 & 0 & -f & 0
\end{array}\right)
$$

At the one-loop level, the self-dual ("SD") EHLs for Scalar and Spinor QED are special cases of (9) and (11). Anticipating the result of the two-loop calculation below, it will be useful here to eliminate the proper-time integral and perform a change of variables from $f$ to $\kappa \equiv m^{2} / 2 e f$. This leads to [8]

$$
\begin{aligned}
& \mathscr{L}_{\text {scal }}^{(1)(\mathrm{SD})}(\kappa) \\
& =\frac{m^{4}}{(4 \pi)^{2}} \frac{1}{\kappa^{2}}\left[-\frac{1}{12} \ln (\kappa)+\zeta^{\prime}(-1)+\Xi(\kappa)\right],
\end{aligned}
$$

where the function $\Xi(x)$ is defined as follows:

$$
\begin{aligned}
\Xi(x) \equiv & \int_{0}^{x} d y \ln \Gamma(y)-x \ln \Gamma(x)+\frac{x^{2}}{2} \ln (x)-\frac{x^{2}}{4} \\
& -\frac{x}{2} .
\end{aligned}
$$

The Spinor EHL in this SD case after renormalization differs from the scalar one only by a trivial global factor of -2 (the reason for the independence of spin is that the Dirac equation in such a background possesses a hidden supersymmetry [28]).

Remarkably, for the SD case it is possible to do all integrals in closed form not only at one loop but even at two loops, in both Scalar and Spinor QED. The results can be written compactly in terms of the digamma function $\psi(x) \equiv$ $\Gamma^{\prime}(x) / \Gamma(x)[8]$ :

$$
\begin{aligned}
& \mathscr{L}^{(2)}(f)=-2 \alpha \frac{m^{4}}{(4 \pi)^{3}} \frac{1}{\kappa^{2}}\left[3 \xi^{2}(\kappa)-\xi^{\prime}(\kappa)\right], \\
& \mathscr{L}_{\text {scal }}^{(2)}(f)=\alpha \frac{m^{4}}{(4 \pi)^{3}} \frac{1}{\kappa^{2}}\left[\frac{3}{2} \xi^{2}(\kappa)-\xi^{\prime}(\kappa)\right] .
\end{aligned}
$$

Here $\kappa \equiv m^{2} / 2 e f$ and

$$
\xi(x) \equiv-x\left(\psi(x)-\ln (x)+\frac{1}{2 x}\right)
$$


(note that $\xi(x)=\Xi^{\prime}(x)$ ). Using the well-known expansion of the digamma function at $x=\infty$ in terms of the Bernoulli numbers

$$
\psi(x) \sim \ln x-\frac{1}{2 x}-\sum_{k=1}^{\infty} \frac{B_{2 k}}{2 k x^{2 k}}
$$

one finds the following closed-form expressions for the oneand two-loop weak-field expansion coefficients $c_{n}^{(1,2)(\mathrm{SD})}$ (we write them down for the Spinor case):

$$
\begin{aligned}
& c_{n}^{(1)(\mathrm{SD})}=-\frac{B_{2 n}}{2 n(2 n-2)}, \\
& c_{n}^{(2)(\mathrm{SD})} \\
& \quad=\frac{1}{(2 \pi)^{2}}\left\{\frac{2 n-3}{2 n-2} B_{2 n-2}+3 \sum_{k=1}^{n-1} \frac{B_{2 k}}{2 k} \frac{B_{2 n-2 k}}{(2 n-2 k)}\right\} .
\end{aligned}
$$

Further, in this self-dual case there is also an analogue of the distinction between a purely magnetic and a purely electric field. For real $f$ the SD EHL turns out to have a weak-field expansion with alternating coefficients, so that it is Borelsummable, and there is no imaginary part. Thus we call this case "magnetic-like." Taking imaginary $f$ removes the alternating sign and creates a pole in the Borel integral, which implies an imaginary part for the EHL. Thus we call this case "electric-like." This imaginary part of the self-dual EHL with complex $f$ is obtained from (35) simply by using the analytic continuation of the digamma function and thus also known in closed form. ways:

Studying the self-dual case turned out to be useful in three

(i) For this case we could verify that the Borel dispersion relation (8) can be used to construct the imaginary part of the EHL from the weak-field expansion even at the two-loop level. That is, the asymptotic threeparameter matching (7) works, and (more nontrivially) the Borel summation procedure does not miss any nonperturbative terms (see [29] for a case where such a thing actually occurred, even at one-loop).

(ii) The AAM exponentiation formula (30) can be generalized to the SD case unchanged, by a simple modification of the worldline instanton to a double circle,

$$
\begin{gathered}
x_{\text {instanton }}(\tau)=\frac{m}{\sqrt{2} e E}\left(\cos \left(\frac{2 \pi \tau}{T}\right), \sin \left(\frac{2 \pi \tau}{T}\right),\right. \\
\left.\cos \left(\frac{2 \pi \tau}{T}\right), \sin \left(\frac{2 \pi \tau}{T}\right)\right) .
\end{gathered}
$$

And the initial step of exponentiation (21) is easy to verify explicitly from (33) and (35). This holds independently of spin.

(iii) The effective action for a self-dual field is unphysical, since such a field cannot be real in Minkowski space. Nevertheless, it still carries information on the physical photon amplitudes; the self-duality condition corresponds precisely to a projection on the "all + " (or "all -") photon amplitudes [30,31]. Thanks to the closed-form expressions (35), even at the two-loop level we are still able to write down a closed-form all$N$ expression for this particular polarization choice:

$$
\begin{aligned}
& \Gamma^{(1)}\left[k_{1}, \varepsilon_{1}^{+} ; \ldots ; k_{N}, \varepsilon_{N}^{+}\right]=-2 \frac{(2 e)^{N}}{(4 \pi)^{2} m^{2 N-4}} c_{N / 2}^{(1)(\mathrm{SD})} \chi_{N}, \\
& \Gamma^{(2)}\left[k_{1}, \varepsilon_{1}^{+} ; \ldots ; k_{N}, \varepsilon_{N}^{+}\right] \\
& \quad=-2 \alpha \pi \frac{(2 e)^{N}}{(4 \pi)^{2} m^{2 N-4}} c_{N / 2}^{(2)(\mathrm{SD})} \chi_{N} .
\end{aligned}
$$

As was mentioned above, here all the dependence on momenta and polarization is absorbed by a unique (independent of loop order) invariant $\chi_{N}$. Using Spinor helicity techniques, this invariant can be constructed explicitly for all $N[10]$.

\section{Synthesis: A Conjecture for the Photon $S$ Matrix}

We are now ready to state a conjecture for the $N$-photon amplitudes at arbitrary loop level $l$ [11]. Consider the $l$-loop correction to the purely electric EHL, and define its weakfield expansion coefficients by

$$
\mathscr{L}^{(l)}(E)=\sum_{n=2}^{\infty} c^{(l)}(n)\left(\frac{e E}{m^{2}}\right)^{2 n}
$$

(note the change of convention with respect to (14)). Assuming that the AAM formula (30) holds and that the Borel dispersion relation (8) works at each loop order, we can conclude that the leading asymptotic factorial growth rate must be the same at each loop order, namely, $\sim \Gamma(2 n-2)$ :

$$
c^{(l)}(n) \stackrel{n \rightarrow \infty}{\sim} c_{\infty}^{(l)} \pi^{-2 n} \Gamma(2 n-2) .
$$

And here $c_{\mathrm{O}}^{(l)}$ relates to the leading Schwinger-exponential at $l$ loops:

$$
\operatorname{Im} \mathscr{L}^{(l)}(E) \stackrel{\beta \rightarrow 0}{\sim} c_{\infty}^{(l)} \mathrm{e}^{-\pi m^{2} / e E} .
$$

At two loops, the numerical calculations of [7] confirm this, but only if physical mass renormalization is used! For generic mass renormalization one finds instead a leading factorial behaviour of $\Gamma(2 n)$, and it is only through a cancellation of this leading order term between the unrenormalized EHL and its mass renormalization counterterm that this leading factorial behaviour gets reduced to the same $\Gamma(2 n-2)$ behaviour as at one loop. At the l-loop level, it is still not difficult to establish the leading factorial growth of the weak-field expansion coefficients before renormalization, which is

$$
c^{(l)}(n) \stackrel{n \rightarrow \infty}{\sim} \Gamma(2 n+2 l-4) .
$$

Thus at higher-loop orders the correctness of the AAM conjecture requires increasingly extensive cancellations in 
the mass renormalization process to cut the leading factorial growth all the way down to $\Gamma(2 n-2)$.

Now let us consider the ratio of the $l$-loop to the oneloop coefficients. Combining (42) and (43) with the AAM conjecture (30), we find at any fixed loop order

$$
\lim _{n \rightarrow \infty} \frac{c^{(l)}(n)}{c^{(1)}(n)}=\frac{c_{\infty}^{(l)}}{c_{\infty}^{(1)}} \stackrel{\text { AAM }}{=} \frac{1}{(l-1) !}(\alpha \pi)^{l-1}
$$

At this stage, let us switch to the self-dual case. This is not essential for our argumentation, but we prefer it for two reasons: first, more is known explicitly about the SD EHL; second, as mentioned above the SD EHL directly translates into one particular helicity component of the $N$-photon amplitude, the one with all helicities equal ("all +" or "all -").

Using our above rule for the conversion of the self-dual weak-field expansion coefficients into the "all +" photon amplitudes, which is independent of the loop order, we get the following statement for the "all +" amplitudes in the limit of large photon number $N=2 n$ :

$$
\begin{aligned}
& \lim _{N \rightarrow \infty} \frac{\Gamma^{(l)}\left[k_{1}, \varepsilon_{1}^{+} ; \ldots ; k_{N}, \varepsilon_{N}^{+}\right]}{\Gamma^{(1)}\left[k_{1}, \varepsilon_{1}^{+} ; \ldots ; k_{N}, \varepsilon_{N}^{+}\right]}=\lim _{N \rightarrow \infty} \frac{\chi_{N} c^{(l)}(N / 2)}{\chi_{N} c^{(1)}(N / 2)} \\
& \quad=\frac{1}{(l-1) !}(\alpha \pi)^{l-1} .
\end{aligned}
$$

Summing this relation over $l$ we get

$$
\lim _{N \rightarrow \infty} \frac{\Gamma^{(\text {total })}\left[k_{1}, \varepsilon_{1}^{+} ; \ldots ; k_{N}, \varepsilon_{N}^{+}\right]}{\Gamma^{(1)}\left[k_{1}, \varepsilon_{1}^{+} ; \ldots ; k_{N}, \varepsilon_{N}^{+}\right]}=e^{\alpha \pi} .
$$

Assuming sufficient uniformity in $l$ of the convergence for $N \rightarrow \infty$, one could now conclude that the amplitude must be analytic in $\alpha$ for some sufficiently large $N$. But analyticity of the complete amplitude can certainly be safely excluded by renormalons and other arguments. Therefore uniformity must fail, and it is easy to see how this comes about diagrammatically. In Figure 5 we show the diagrams contributing to the EHL up to four loops, not showing the external legs.

In the worldline instanton based derivation of the AAM conjecture (30), only quenched diagrams contribute to the weak-field limit of the imaginary part of the electric EHL; thus this must also be true for the leading asymptotic terms in the large $N$ expansion of the weak-field expansion coefficients; nonquenched diagrams must get suppressed for $N \rightarrow \infty$. However, the number of such diagrams is strongly growing with the loop order, so that the process of the suppression of the nonquenched contributions by increasing $N$ should slow down with increasing $l$. This provides a good reason for uniformity to fail for the whole amplitude, but there is no obvious reason to expect such a nonuniformity if one stays inside the class of quenched diagrams from the beginning. This led Dunne and one of the authors in 2006 [11] to conjecture that perturbation theory converges for the QED photon amplitudes in the one-electron-loop approximation. If true, this would imply enormous cancellations between Feynman diagrams, presumably due to gauge invariance.

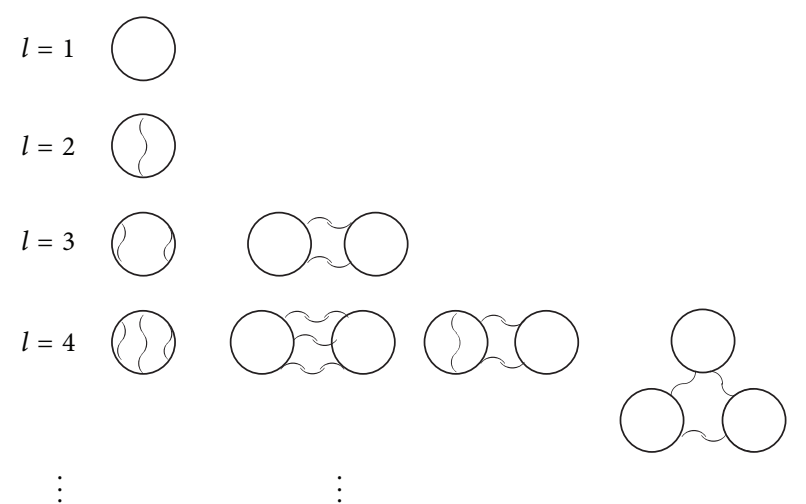

FIgURE 5: Diagrams contributing to the EHL up to four loops (external legs not shown).

Only afterwards we learned that, as mentioned in the beginning, Cvitanovic [5] had conjectured the analogous statement for the electron $g-2$ factor,

\section{Three Predictions for the Three-Loop EHL}

To either disprove or further corroborate this conjecture, a calculation of the EHL at the three-loop level is called for. We would like to see the following three things happen:

(1) We should see the next term of the exponentiation:

$$
\lim _{n \rightarrow \infty} \frac{c^{(3)}(n)}{c^{(1)}(n)}=\frac{1}{2}(\alpha \pi)^{2} .
$$

(2) At three loops there is already a nonquenched contribution, and it should be suppressed in the large $N$ limit.

(3) The convergence of $c^{(3)}(n) / c^{(1)}(n)$ should not be slower than the one of $c^{(2)}(n) / c^{(1)}(n)$ when only quenched diagrams are taken.

However, a calculation of the three-loop EHL in $D=4$ seems presently technically out of reach.

\section{QED in 1 + 1 Dimensions}

The proper-time representation of the one-loop EHL is essentially independent of dimension. In 2008 Krasnansky [32] studied the Scalar EHL in various dimensions also at two loops and found, in particular, the rather surprising fact that the Scalar EHL in $1+1$ dimensions even at two loops has a structure almost identical to the one of the self-dual Scalar EHLs in $3+1$ dimensions. Let us contrast the two cases: above we wrote down the self-dual field strength tensor for $D=4$ :

$$
F=\left(\begin{array}{cccc}
0 & f & 0 & 0 \\
-f & 0 & 0 & 0 \\
0 & 0 & 0 & f \\
0 & 0 & -f & 0
\end{array}\right) .
$$


We also gave the Scalar EHL for this background:

$$
\begin{aligned}
\mathscr{L}_{\text {scal }}^{(2)(4 D)}(\kappa) & =\alpha \frac{m^{4}}{(4 \pi)^{3}} \frac{1}{\kappa^{2}}\left[\frac{3}{2} \xi^{2}-\xi^{\prime}\right], \\
\xi(\kappa) & =-\kappa\left(\psi(\kappa)-\ln (\kappa)+\frac{1}{2 \kappa}\right) .
\end{aligned}
$$

In $2 \mathrm{D}$ the field strength tensor is $F=\left(\begin{array}{cc}0 & f \\ -f & 0\end{array}\right)$, and the twoloop Scalar QED EHL comes out as [32]

$$
\begin{aligned}
\mathscr{L}_{\text {scal }}^{(2)(2 D)}(\kappa) & =-\frac{e^{2}}{32 \pi^{2}}\left[\xi_{2 D}^{2}-4 \kappa \xi_{2 D}^{\prime}\right], \\
\xi_{2 D} & =-\left(\psi\left(\kappa+\frac{1}{2}\right)-\ln (\kappa)\right) .
\end{aligned}
$$

Since higher-loop calculations are somewhat easier in two dimensions, this suggests using the $2 \mathrm{D}$ case as a toy model for studying the AAM conjecture. An effort along these lines was started in [13], however switching from Scalar to Spinor QED. Here we derived an analogue of the AAM conjecture in 2D, also using the worldline instanton approach, and established the correspondences between the $4 \mathrm{D}$ and $2 \mathrm{D}$ cases shown in Box 1.

There are two essential differences. First, in 2D the fine structure constant $\widetilde{\alpha}$ is not dimensionless. Thus the exponent of the AAM formula (rhs of third line) here involves also a factor of $\kappa^{2}$, which in the formula for the asymptotic behaviour of the weak-field expansion coefficients (rhs of fourth line) leads to a shift in the argument between the $l$-loop and the one-loop coefficients. Thus in 2D the leading asymptotic growth of the coefficients increases with increasing loop order, as it does in the $4 \mathrm{D}$ case before mass renormalization, and correspondingly it can be shown that the contributions to the EHL from mass renormalization are asymptotically subleading and thus irrelevant for our purposes (although the fermion propagator in 2D does not have UV divergences, mass renormalization is still a quite nontrivial issue; see [33] and refs. therein). Presumably this relates to the fact that QED in $2 \mathrm{D}$ is confining.

In any case, all three of our three-loop predictions above have an analogue in the $2 \mathrm{D}$ case. In [13] we also obtained the following formulas for the one- and two-loop EHLs in 2D Spinor QED:

$$
\begin{aligned}
& \mathscr{L}^{(1)}(\kappa) \\
& =-\frac{m^{2}}{4 \pi} \frac{1}{\kappa}\left[\ln \Gamma(\kappa)-\kappa(\ln \kappa-1)+\frac{1}{2} \ln \left(\frac{\kappa}{2 \pi}\right)\right], \\
& \mathscr{L}^{(2)}(f) \\
& =\frac{m^{2}}{4 \pi} \frac{\widetilde{\alpha}}{4}\left[\widetilde{\psi}(\kappa)+\kappa \widetilde{\psi}^{\prime}(\kappa)+\ln \left(\lambda_{0} m^{2}\right)+\gamma+2\right],
\end{aligned}
$$

where we have now abbreviated

$$
\widetilde{\psi}(x) \equiv \psi(x)-\ln x+\frac{1}{2 x} .
$$

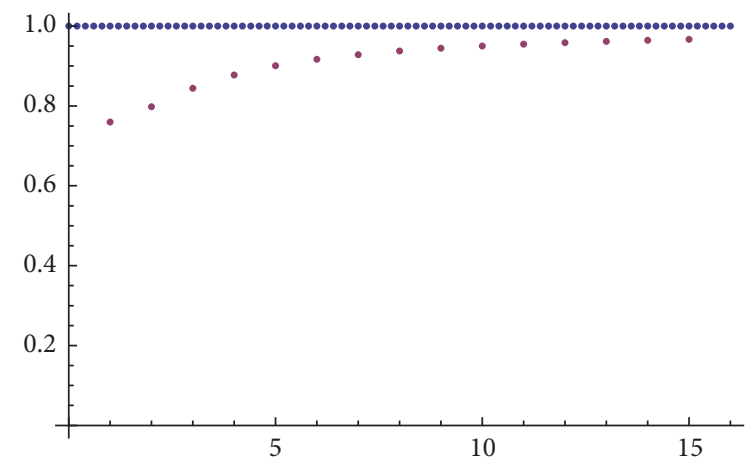

FIGURE 6: Convergence of $c_{2 D}^{(2)}(n) / c_{2 D}^{(1)}(n+1)$ to the AAM prediction (normalized such that the limit is unity).

Comparing with the Scalar QED result (51), we see that the Spinor QED one is significantly simpler, as it involves the digamma function only linearly. This is another surprise, since in $4 \mathrm{D}$ the Scalar and Spinor EHLs do not show structural differences.

Remarkably, the two-loop EHL can (up to an irrelevant constant) even be written in terms of derivatives of the oneloop EHL:

$$
\mathscr{L}^{(2)}(f)=-\frac{\tilde{\alpha}}{4}\left(m^{2} \frac{\partial}{\partial m^{2}}\right)^{2} \mathscr{L}^{(1)}(f)
$$

From (52) we find for the one- and two-loop weak-field expansion coefficients

$$
\begin{aligned}
c_{2 D}^{(1)}(n) & =(-1)^{n+1} \frac{B_{2 n}}{4 n(2 n-1)}, \\
c_{2 D}^{(2)}(n) & =(-1)^{n+1} \frac{\widetilde{\alpha}}{8} \frac{2 n-1}{2 n} B_{2 n} .
\end{aligned}
$$

From this we can, using properties of the Bernoulli numbers, easily show that

$$
\lim _{n \rightarrow \infty} \frac{c_{2 D}^{(2)}(n)}{c_{2 D}^{(1)}(n+1)}=\widetilde{\alpha} \pi^{2} .
$$

This verifies the 2D AAM-like formula of Box 1 at the linearized level.

In Figure 6 we show the convergence to the asymptotic limit, which is rather rapid.

Even in the 2D case, the calculation of the three-loop EHL turned out to be a formidable task, and it is only very recently that we were able to obtain it in a form suitable for computing a sufficient number of the weak-field expansion coefficients $[14,15]$.

At three loops, we have the three Feynman diagrams shown in Figure 7.

Here the solid lines denote the electron propagator in the constant field. A and B are quenched; C is nonquenched. 


$$
\begin{aligned}
& \text { 4D QED } \longleftrightarrow \text { 2D QED } \\
& \alpha=\frac{e^{2}}{4 \pi} \longleftrightarrow \widetilde{\alpha}=\frac{2 e^{2}}{\pi m^{2}} \\
& \operatorname{Im} \Gamma^{D=4} \sim \mathrm{e}^{-m^{2} \pi / e E+\alpha \pi} \longleftrightarrow \operatorname{Im} \Gamma^{D=2} \sim \mathrm{e}^{-m^{2} \pi / e E+\widetilde{\alpha} \pi^{2} \kappa^{2}} \\
& \lim _{n \rightarrow \infty} \frac{c_{4 D}^{(l)}(n)}{c_{4 D}^{(1)}(n)}=\frac{(\alpha \pi)^{l-1}}{(l-1) !} \longleftrightarrow \lim _{n \rightarrow \infty} \frac{c_{2 D}^{(l)}(n)}{c_{2 D}^{(1)}(n+l-1)}=\frac{\left(\widetilde{\alpha} \pi^{2}\right)^{l-1}}{(l-1) !}
\end{aligned}
$$

Mass renormalization essential $\longleftrightarrow$ Mass renormalization irrelevant

Box 1: Correspondences between the 4D and 2D cases.

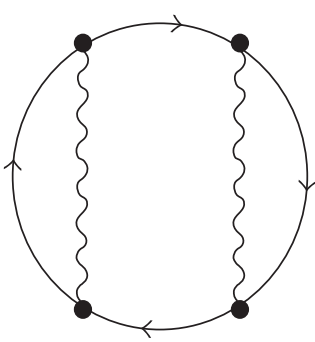

A

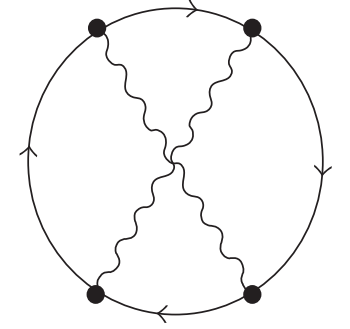

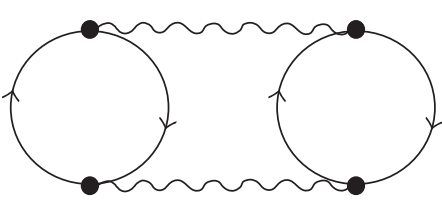

C

Figure 7: Three-loop Feynman diagrams.

The last one is by far the easiest one, and it is straightforward to obtain for it the following compact integral representation:

$$
\begin{aligned}
& \mathscr{L}^{3 C}(f)=\frac{e^{3}}{16 \pi^{3} f} \int_{0}^{\infty} d z d z^{\prime} d \widehat{z} d z^{\prime \prime} \\
& \cdot \frac{\sinh z \sinh z^{\prime} \sinh \widehat{z} \sinh z^{\prime \prime}}{\left[\sinh \left(z+z^{\prime}\right) \sinh \left(\widehat{z}+z^{\prime \prime}\right)\right]^{2}} \\
& \cdot \frac{\mathrm{e}^{-2 \kappa\left(z+z^{\prime}+\widehat{z}+z^{\prime \prime}\right)}}{\sinh z \sinh z^{\prime} \sinh \left(\widehat{z}+z^{\prime \prime}\right)+\sinh \widehat{z} \sinh z^{\prime \prime} \sinh \left(z+z^{\prime}\right)} .
\end{aligned}
$$

This representation turned out to be quite adequate for a numerical calculation of the first 9 weak-field expansion coefficients $c_{C}^{(3)}(n)$ of diagram C. In Figure 8 we use these nine coefficients to show that this unquenched contribution is indeed asymptotically subleading.

This settles point 2 of our wish list above!

Diagrams A and B are much more difficult, but the use of the "traceless gauge" choice $\lambda=2$ led to simplifications and in particular to manifesting IR finiteness term by term. We managed to compute the first coefficient analytically

$$
c_{A+B}^{3}(0)=\left(-\frac{3}{2}+\frac{7}{4} \zeta(3)\right) \frac{\widetilde{\alpha}^{2}}{64}
$$

and five more coefficients numerically. Using these to plot the ratio $\left(c_{A+B}^{(3)}(n) / c_{2 D}^{(1)}(n+2)\right) /\left(\left(\widetilde{\alpha} \pi^{2}\right)^{2} / 2\right)$ we get Figure 9.

Thus we are falling even below the asymptotic prediction!

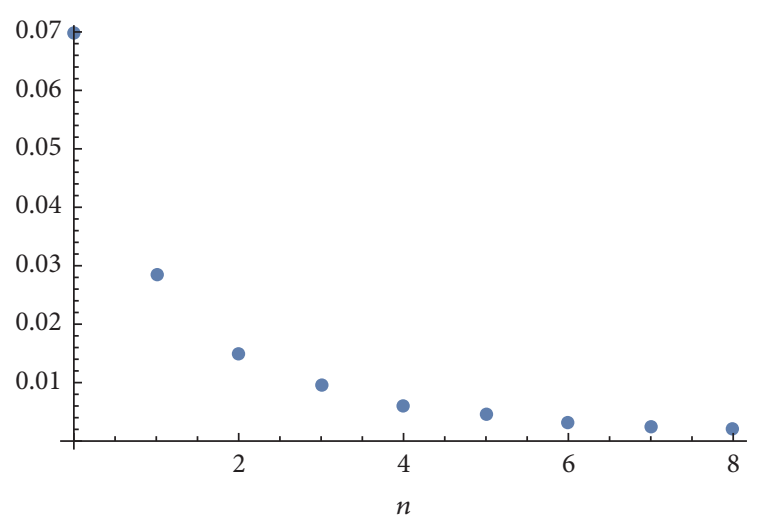

Figure 8: The ratio $\left(c_{C}^{(3)}(n) / c_{2 D}^{(1)}(n+2)\right) /\left(\left(\widetilde{\alpha} \pi^{2}\right)^{2} / 2\right)$ for $n=0, \ldots, 8$.

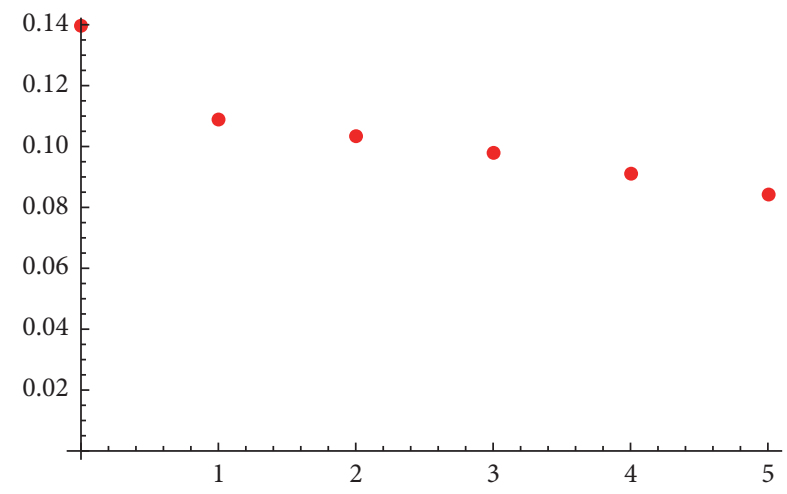

Figure 9: The ratio $\left(c_{A+B}^{(3)}(n) / c_{2 D}^{(1)}(n+2)\right) /\left(\left(\widetilde{\alpha} \pi^{2}\right)^{2} / 2\right)$ for $n=0, \ldots, 5$. 


\section{Conclusions and Outlook}

Let us summarize:

(i) We have presented here first results of a calculation of the three-loop 2D EHL. This is the first calculation of a three-loop effective Lagrangian in QED.

(ii) Although so far we have been able to compute only six coefficients of the weak-field expansion (we should be able to obtain a few more) it seems already very likely that the analogue of the AAM conjecture fails in 2D QED. This would throw also serious doubts on the validity of the $4 \mathrm{D}$ AAM conjecture.

(iii) However, since the coefficient ratios fall below, rather than above, the asymptotic prediction, the riddle of the unreasonable smallness of loop corrections remains. Presumably the worldline instanton approach captures some valid information on large-scale cancellations between Feynman diagrams but needs refinement beyond two loops.

(iv) We have also made an effort to make the point that the QED photon amplitudes in the limit of low energy and large number of photons are very natural objects for a study of the asymptotic properties of the QED perturbation series.

(v) It should also have become clear that physical mass renormalization is essential for asymptotic estimates in QED! Unless mass renormalization is done physically, QED perturbation theory will break down already at the two-loop level, because the two-loop contribution to any helicity component of the $N$ photon amplitude will, at least in the low-energy limit, dominate over the one-loop one for sufficiently large $N$. This implies, in particular, that approaches to the study of the large-order behaviour of the QED perturbation series that are indifferent to the issue of physical mass renormalization ought to be viewed with caution.

(vi) As an aside, it would be interesting to study also the QCD $N$-gluon amplitudes for large $N$ from the point of view of mass renormalization.

\section{Conflicts of Interest}

The authors declare that there are no conflicts of interest regarding the publication of this paper.

\section{References}

[1] F. J. Dyson, "Divergence of perturbation theory in quantum electrodynamics," Physical Review, vol. 85, no. 4, pp. 631-632, 1952.

[2] C. A. Hurst, "An example of a divergent perturbation expansion in field theory," vol. 48, pp. 625-639, 1952.

[3] G. 't Hooft, "Can we make sense out of 'quantum chromodynamics'?" in The Whys of Subnuclear Physics, A. Zichichi, Ed., Plenum, New York, NY, USA, 1978, Reprinted in: G. 't
Hooft, Under the Spell of The Gauge Principle, World Scientific, Singapore, 1994.

[4] J. G. Russo, "A note on perturbation series in supersymmetric gauge theories," Journal of High Energy Physics, vol. 2012, p. 38, 2012.

[5] P. Cvitanovic, "Asymptotic estimates and gauge invariance," Nuclear Physics B, vol. 127, pp. 176-188, 1977.

[6] P. Cvitanovic and T. Kinoshita, "Sixth-order magnetic moment of the electron," Physical Review D, vol. 10, artice 4007, 1974.

[7] G. V. Dunne and C. Schubert, "Two-loop Euler-Heisenberg QED pair-production rate," Nuclear Physics B, vol. 564, Article ID 9907190, p. 591, 2000.

[8] G. V. Dunne and C. Schubert, "Two-loop self-dual EulerHeisenberg Lagrangians (I): real part and helicity amplitudes," Journal of High Energy Physics, vol. 2002, article 0208, p. 53, 2002.

[9] G. V. Dunne and C. Schubert, "Two-loop self-dual EulerHeisenberg Lagrangians (II): imaginary part and Borel analysis," Journal of High Energy Physics, vol. 2002, article 0206, 2002.

[10] L. C. Martin, C. Schubert, and V. M. Villanueva Sandoval, "On the low-energy limit of the QED N-photon amplitudes," Nuclear Physics B, vol. 668, no. 1-2, Article ID 0301022, pp. 334-335, 2003.

[11] G. V. Dunne and C. Schubert, "Multiloop information from the QED effective Lagrangian,” Journal of Physics. Conference Series, vol. 37, pp. 59-72, 2006.

[12] G. V. Dunne, A. Huet, D. Rivera, and C. Schubert, "Closedform weak-field expansion of two-loop Euler-Heisenberg lagrangians," Journal of High Energy Physics, vol. 2006, 2006.

[13] I. Huet, D. G. C. McKeon, and C. Schubert, "Euler-Heisenberg lagrangians and asymptotic analysis in 1+1 QED. Part I: twoloop," Journal of High Energy Physics, vol. 2010, article 1012, p. 36, 2010.

[14] I. Huet, M. R. De Traubenberg, and C. Schubert, "The EulerHeisenberg Lagrangian beyond one loop," in Proceedings of the Eleventh Conference on Quantum Field Theory under The Influence of External Conditions (QFEXT '11), vol. 14, pp. 383393,2012

[15] I. Huet, M. Rausch de Traubenberg, and C. Schuber, “Threeloop Euler-Heisenberg Lagrangian in 1+1 QED," In preparation.

[16] W. Heisenberg and H. Euler, "Folgerungen aus der diracschen theorie des positrons," Zeitschrift für Physik, vol. 98, no. 11-12, pp. 714-732, 1936.

[17] V. Weisskopf, Kong. Dans. Vid. Selsk. Math-fys. Medd. XIV No. 6 (1936), Quantum Electrodynamics, Dover, New York, NY, USA, 1958.

[18] F. Sauter, "Über das Verhalten eines Elektrons im homogenen elektrischen Feld nach der relativistischen Theorie Diracs," Zeitschrift für Physik, vol. 69, no. 11-12, pp. 742-764, 1931.

[19] J. Schwinger, "On gauge invariance and vacuum polarization," Physical Review, vol. 82, pp. 664-679, 1951.

[20] V. I. Ritus, "Lagrangian of an intense electromagnetic field and quantum electrodynamics at short distances," Journal of Experimental and Theoretical Physics, vol. 69, p. 1517, 1975, [Sov. Phys. JETP 42 (1975) 774].

[21] V. I. Ritus, "On the relation between the quantum electrodynamics of an intense field and the quantumelectrodynamics at small distances," Zh.Eksp.Teor.Fiz, vol. 73, pp. 807-821, 1977 (Russian).

[22] W. Dittrich and M. Reuter, Effective Lagrangians in Quantum Electrodynamics, Springer, Berlin, Germany, 1985. 
[23] M. Reuter, M. G. Schmidt, and C. Schubert, "Constant external fields in gauge theory and the spin 0,1,2,1 path integrals," Annals of Physics, vol. 259, no. 2, pp. 313-365, 1997.

[24] S. L. Lebedev and V. I. Ritus, "Virial representation of the imaginary part of the lagrange function of the electromagnetic field," Zh.Eksp.Teor.Fiz, vol. 86, pp. 408-421, 1984.

[25] V. I. Ritus, "Method of eigenfunctions and mass operator in quantum electrodynamics of a constant field," Zh.Eksp.Teor.Fiz, vol. 75, pp. 1560-1583, 1978.

[26] I. K. Affleck, O. Alvarez, and N. S. Manton, "Pair production at strong coupling in weak external fields," Nuclear Physics, Section $B$, vol. 197, no. 3, pp. 509-519, 1982.

[27] R. P. Feynman, "Mathematical formulation of the quantum theory of electromagnetic interaction," Physical Review A, vol. 80, p. 440, 1950.

[28] A. D’Adda and P. Di Vecchia, "Supersymmetry and instantons," Physics Letters B, vol. 73, no. 2, pp. 162-166, 1978.

[29] A. Das and G. V. Dunne, "Large-order perturbation theory and de Sitter/anti de Sitter effective actions," Physical Review. D. Third Series, vol. 74, no. 4, 044029, 9 pages, 2006.

[30] M. J. Duff and C. J. Isham, "Self-duality, helicity, and supersymmetry: The scattering of light by light," Physics Letters B, vol. 86, no. 2, pp. 157-160, 1979.

[31] M. J. Duff and C. J. Isham, "Self-duality, helicity and coherent states in nonabelian gauge theories," Nuclear Physics. B, vol. 162, no. 2, pp. 271-284, 1980.

[32] M. Krasnansky, "Two-loop vacuum diagrams in background field and the Heisenberg-Euler effective action," International Journal of Modern Physics A. Particles and Fields. Gravitation. Cosmology, vol. 23, no. 32, pp. 5201-5215, 2008.

[33] A. Das, J. Frenkel, C. Schubert, and B. Phys. Lett, "Infrared divergences, mass shell singularities and gauge dependence of the dynamical fermion mass," Physics Letters B, vol. 720, no. 45, pp. 414-418, 2013. 

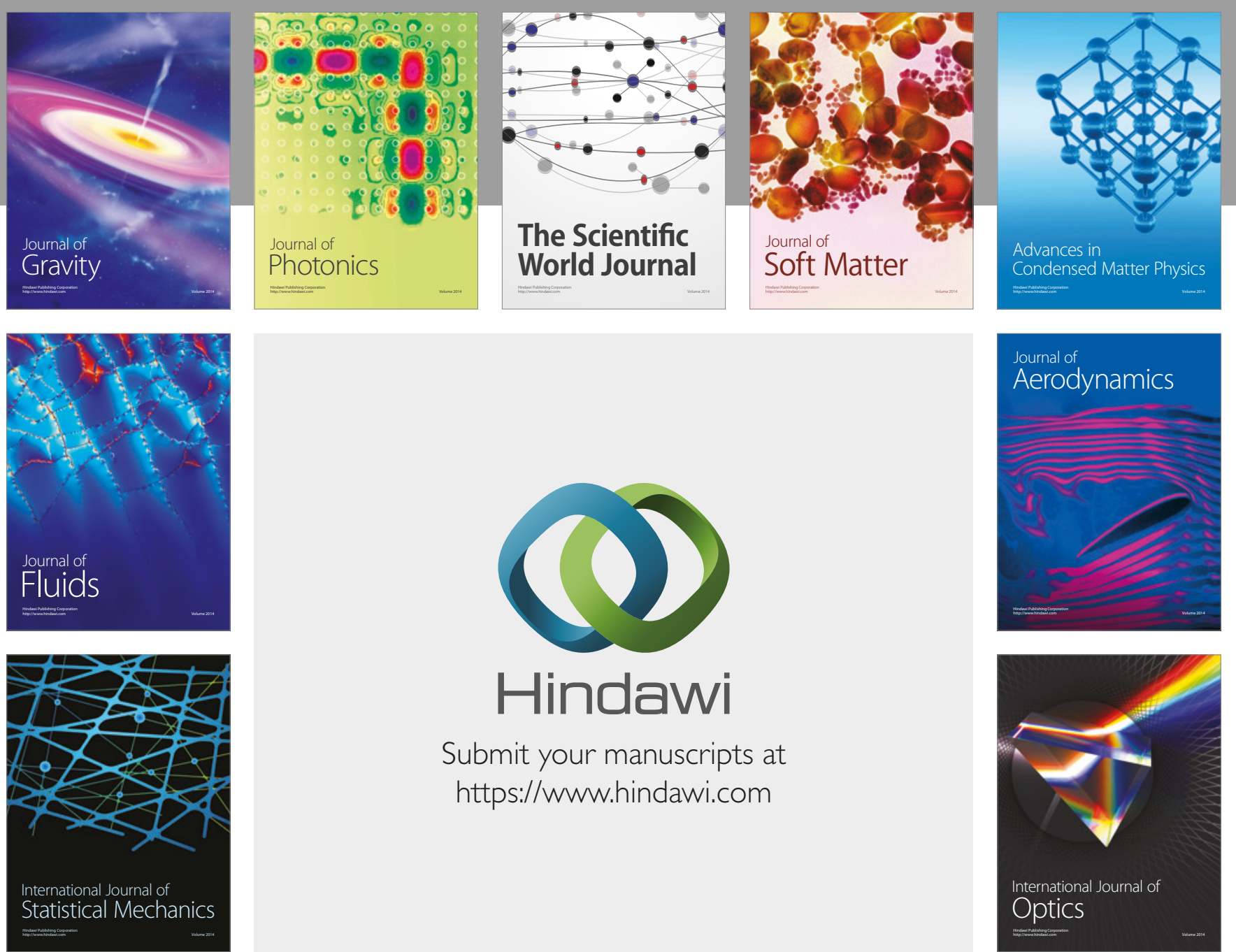

Submit your manuscripts at

https://www.hindawi.com
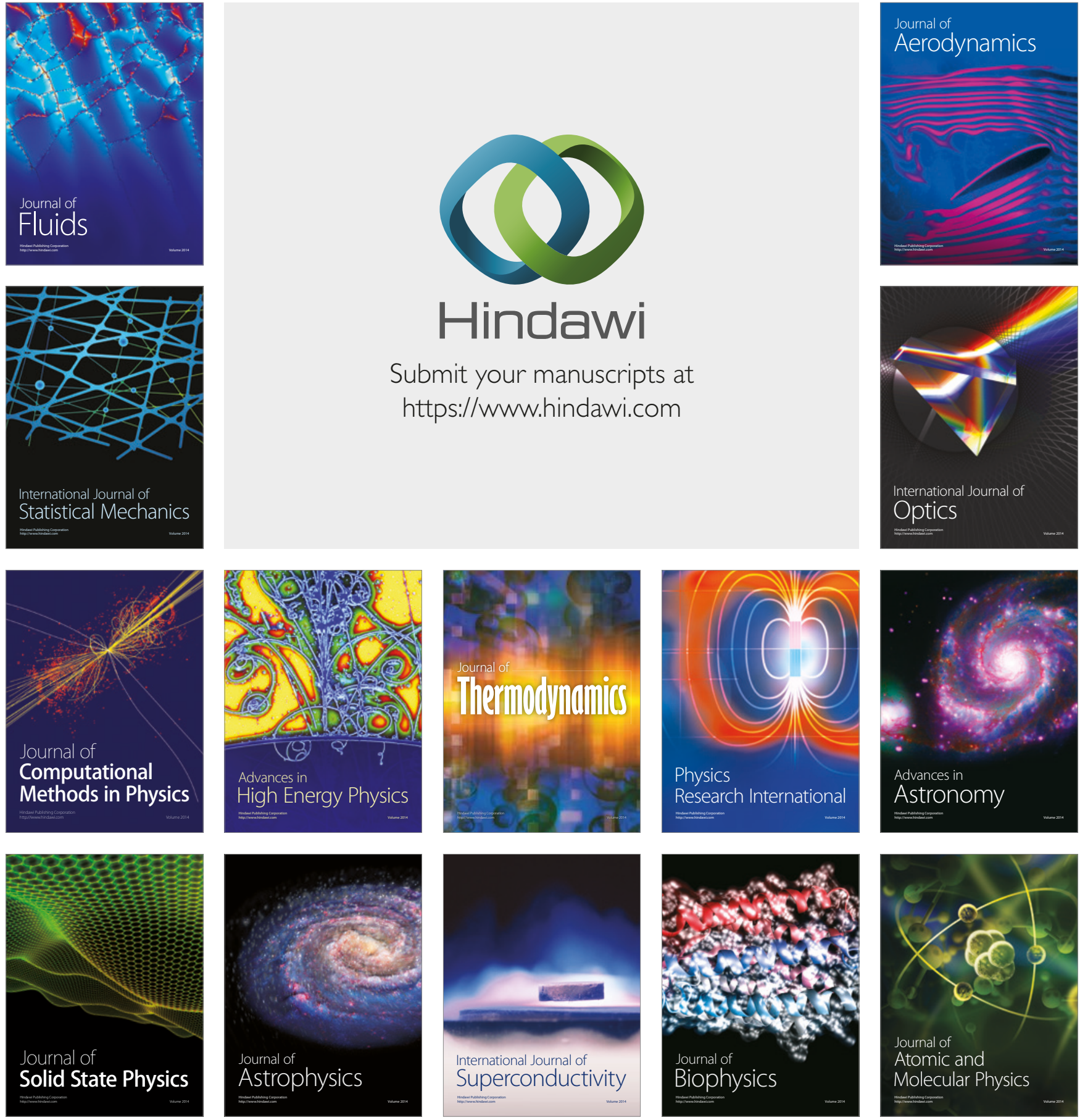